\title{
Property Modifiable Discreet Active Landmarks
}

\author{
Tetsuo Tomizawa, Yoichi Morales Saiki, Akihisa Ohya, and Shin'ichi Yuya
}

\begin{abstract}
This paper explains the design and implementation of a discreet landmark capable of modifying its properties in order to be able to differentiate itself from its surrounding environment. In this case, differently from common devices, our landmark does not make use of neither light, wave reflection, or radio frequencies, but it makes use of temperature properties of a peltier device which is able to increase or decrease its temperature. By having different characteristics from its surroundings, in this case a temperature landmark can be robustly found and tracked in complex indoor environments using thermo infrared cameras. The proposed system composition and configuration, landmark finding, extraction and tracking methods are also explained and effectiveness of this system is described and evaluated.
\end{abstract}

\section{INTRODUCTION}

Autonomous mobile robots navigating in indoor environments, commonly make use of landmarks to determine its position and correct dead reckoning's accumulated error. Indoor robots usually use cameras and laser sensors to localize, identify and measure landmarks in the environment, however, in environments where light conditions continually change introducing noisy measurements and where multiple objects cause occlusion between landmarks and robots, difficulty to find landmarks increases considerably. These kinds of factors limit environments where autonomous robots can navigate.

Laser range finders are commonly used in indoor robot navigation for environment scanning and map matching. Walls and corners are usually used as landmarks in wide rooms and hallways [1][2]. On the other hand, for more complex environments such as homes, offices and supermarkets where there are multiple objects, it is difficult to accurately find and extract landmarks. In these cases robots have to rely on dead reckoning methods such as odometry and inertial navigation with the problem of having unbounded accumulation of posture errors as time passes by. Even if robot is able to find a landmark, because it has accumulated error it is difficult to identify it between other landmarks and accurately correct its position and orientation.

It is desirable that landmarks have unique characteristics different from its surroundings, but in dynamic complex environments it is not an easy task to achieve. In order to be able to recognize robustly a particular object in these conditions, we propose and developed a device that actively can change

T. Tomizawa is member of Ubiquitous Function Research Group, Intelligent Systems Institute, National Institute of Advanced Industrial Science and Technology, Tsukuba, 305-8568, Japan t.tomizawa@aist.go.jp

Y. Morales Saiki, A. Ohya and S. Yuta are members of Intelligent Robot Laboratory, Graduate School of Systems and Information Engineering, University of Tsukuba, Tsukuba, 305-8573, Japan \{yoichi, ohya, yuta\}eroboken.cs.tsukuba.ac.jp

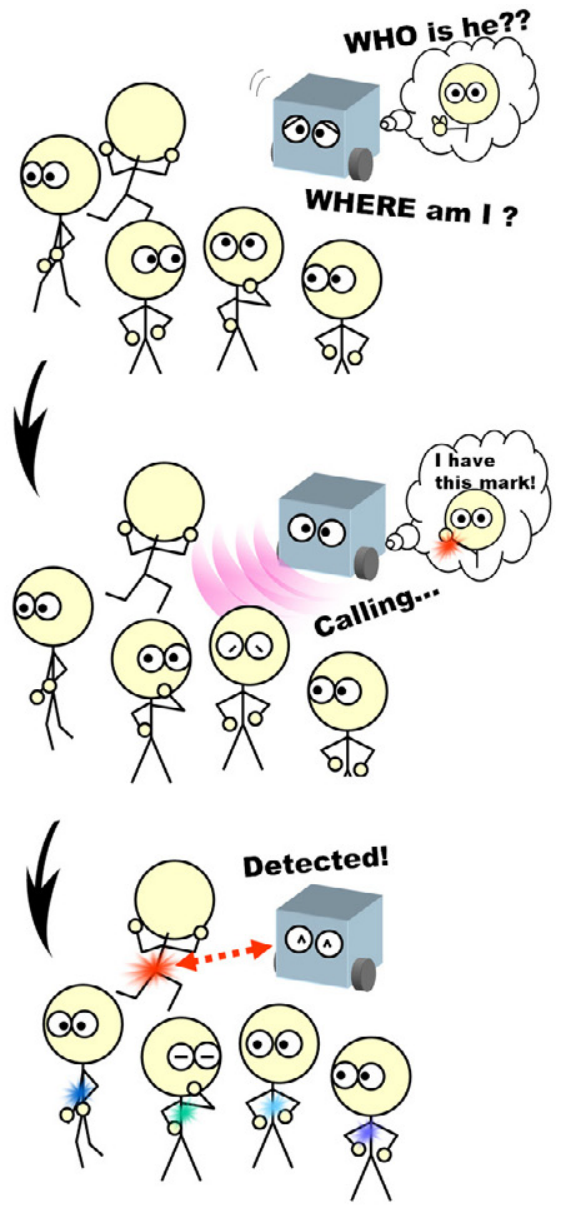

Fig. 1. Image of Human Detection System using an Active Landmark.

its own features according to surrounding conditions (see Fig.1).

By using a self-feature changing device, a landmark can be used in two ways:

- To change itself to have peculiar characteristics in a determined environment

- To merge into the environment

In the first case, the mark can change its properties to allow itself to be found by a sensor. In the second case, landmark can modify its characteristics to hide itself in the environment to allow other landmarks to be found without interfering.

Besides the possible uses explained above, in the case these kind of devices are placed in common indoor environments, it is desirable that they do not get into the sight of view of persons and that they can change its properties 
by itself without people around noticing these changes, this is the landmarks should be as discreet as possible.

\section{RELATED WORKS}

Commonly, for mobile robot localization, usual available objects such as walls and desks are used as landmarks which can be easily found by Laser Range Finders and cameras extracting straight lines from their surfaces. On the other hand, recent development of networking techniques and robotics technologies made available to place distributed sensors and actuators in the environment, giving them the capability of not only be a passive element in robot localization, but to interact with mobile robots as explained in [3]. By giving physical environments this capability they can provide mobile robots useful information which would be difficult to extract on their own. The concept of distributed sensors and actuators is an actual topic widely being investigated. Next we present some examples of different types of landmarks.

\section{A. Optical Landmarks}

A common optic landmark is an infrared LED transceiver placed on a roof, wall or person that emits light in order to be found. Infrared rays have the advantage that are not visible to human eyes and can be used as invisible landmarks. If it emits light in a determined frequency, it can easily be detected and demodulated with a camera with filter. After image processing and coordinate conversion, landmark can be extracted and its position calculated.

As concrete examples, it can be mentioned the system in [4] which consist of a mobile robot with a LED on it and a camera on roof to localize robot and correct its position. Another example is the system in [5] in which LED modules are placed on the environment and mobile robot with cameras localize them. In [6], a mobile robot following a blinking LED module carried by a person is explained. In the previously explained examples, recognition of LEDs using a camera with filter is achieved, however, in bright places when sunlight is reflected to the camera it is considerable difficult to find the marks. Moreover, when there are several devices emitting light, it is almost impossible to be able to differentiate each light emitting device from each other.

\section{B. Ultrasonic Wave Landmarks}

Ultrasonic sensors emitting and receiving ultrasonic waves can measure the relative distance to an object[6]. In an environment with multiple ultrasonic landmarks emitting modulated signals a rover receives these signals demodulating them to find the emitter of the wave, if it finds it, it send a signal back ("Eco Back") to the emitting transponder.

Common Ultrasonic Sensors measure the time of flight (TOF) of the ultrasonic wave to calculate distance. Each device has a receiver and transmitter transducer. By receiving wave signals in small periods of time, rover can measure where they come from. The technical difficulty with ultrasonic waves is that they have narrow frequency band and when receiving signals from several transmitters, it is considerably difficult to recognize the origin of each emitter.

\section{RFID Landmarks}

By reading an object's IC tag with an IC tag reader it is possible to load from its data base information such as name and coordinates [7]. If an IC-tag is embedded on a wall, roof or floor, when the tag sensor passes through its proximity, it will be ale to identify the objects around it and to estimate its own position.

\section{GPS}

In GPS system, pseudoranges (time of flight of waves from satellites to land rover receiver) are used obtain absolute position. This system can only be used in outdoor environments where there are not tall obstacles around and there is free line of view to satellites [8]. On the other hand, GPS can not be used in indoor environments because land rover can not receive satellite signals. In indoor environments, pseudollites [9] can be used using a similar principle to the GPS system, yet, the system has a high cost and it suffers from signal multipath effects.

\section{E. Discreet Passive Markers for Localization}

In previous related works explained, active landmarks were used, however in [10], a passive landmark system is explained. In this system reflexive sheets were placed on the roof and walls, a robot emits infrared ray which after reflexion are measured back.

\section{TEMPERATURE LANDMARK SYSTEM}

\section{A. Changeable Characteristic Selection}

The main objective of this research is to develop a mark able to change its characteristics according to the surrounding environment. First we made an analysis of what characteristics could be easily changed in a landmark according to surrounding complex environments. Some characteristics can be mentioned:

- Color/luminosity intensity

- Form

- Temperature

In the case of color there are devices that can change its luminosity such as LEDs, displays and liquid crystal films. These devices can easily change its color and be found by a common camera. Complexity arises when light conditions change in the environment because robustly finding a determined color is difficult. Moreover, complex environments such as homes and offices have multiple colorful objects, so it is not easy to find an unique determined color.

In the second case, object form transformation can easily be done by small motors, air actuators or shape-memory metals. Edges and angles of certain objects can be found and extracted by LRF and image processing techniques. However, to recognize objects high accuracy sensors are needed and form modifiable objects would need to be big enough to be found. 
In the two previously explained methods, object characteristics can be easily changed but modifications would be noticeable for humans. For example color change would be noticeable and attract people's attention and in body transformation if the object is too big could even be dangerous for people around.

For these reasons, for this research we decided to focus on object temperature. While temperature mark is not directly touched, this mark could not be noticed by persons in the surrounding areas. Every objects radiate infrared rays according to their body temperature. Objects with low temperature irradiate weak infrared rays from its surface, on the other hand, as body temperature increases, radiated infrared strength proportionally increase. Therefore by using this principle, if we now the intensity of infrared rays in the environment, we can measure the temperature of the surroundings.

\section{B. Basic Strategy}

Our system consists of a "Temperature Landmark" and a thermo infrared camera. The configuration of each element is presented in figure 2 and figure 3. The basic strategy for recognizing and finding landmarks is explained as follows:

1) Thermo infrared camera indicates temperature landmark module a determined temperature in which it should change

2) Infrared camera searches for landmark in the previously indicated temperature

3) When there are objects with similar temperature to the selected one, then our landmark is set to change its temperature (this temperature is found analyzing temperature histogram in camera to find less common temperatures)

Wireless communication between landmark and camera allows camera module to change landmark's temperature, greatly expanding areas in which our proposed system could be employed.

\section{System CONFIGURATION}

\section{A. Specifications}

Our system consists of a thermo infrared camera attached on a mobile robot that moves around its environment looking for the temperature landmark. The range in which camera can find the landmark goes from 1 to 3 meters.

\section{B. Temperature Landmark}

1) Hardware Configuration: They key piece in this system is a peltier device from S.T.S. serial number T151-40031S (Fig.4(c)). This device can change its temperature from $0^{\circ}$ to $90^{\circ}$ Celsius according to polarization of its terminals. Peltier device area is small, so one side is attached to a heat radiator to increase its temperature visibility area.

A one-chip microprocessor model c8051 from Cygnal Technologies (Fig.4(a)) is used as landmark temperature controller. Peltier device has the property to increase and

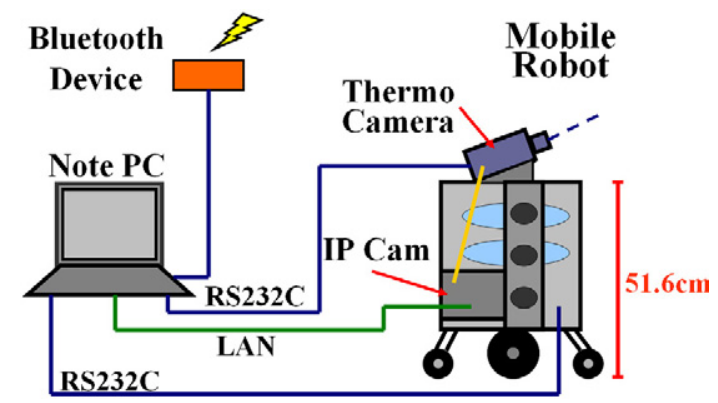

Fig. 2. Structure of the Termo-Detector System.

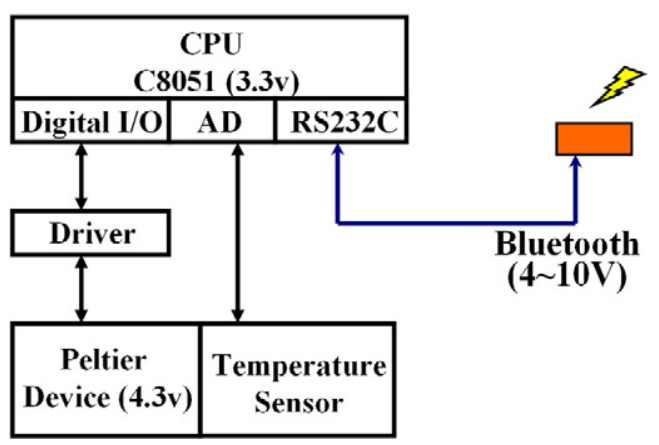

Fig. 3. Structure of the Thermo-Landmark.

decrease its temperature depending on how polarization in its terminals is produced. Temperature is controlled according in how much current is fed to the device; in order to achieve this we use an iMDs03 motor driver (Fig.4(d)) from iXs Research Corporation to feed the landmark by PWM.

Communication between landmark and user PC is achieved using a bluetooth wireless module from Best Technology (Fig.4(b)). This device has a maximum range of 10 meters and a communication speed of $723 \mathrm{kbps}$. Landmark module comprised of micro controller, peltier device and bluetooth module is shown in Fig.5.

2) Peltier Device Temperature Properties: Peltier device has attached to it an electric temperature sensor which senses its body temperature. Feedback temperature control is done by reading an electric thermometer and feeding current to peltier device by PWM pulses. Fig.6 shows an example of feedback control temperature in Celsius degrees vs. time. As it can be seen in the graph, temperature changes at a rate of 10 degrees per second. In our system, we used only one peltier device but if a faster change rate is needed multiple peltier devices can be used.

\section{Infrared Temperature Camera}

We use an infrared thermograph camera from Nippon Avionics model TVS-600 (dimensions: $115 \mathrm{~mm} x 217 \mathrm{~mm} x$ $142 \mathrm{~mm}$ ). This camera has $35 \mathrm{~mm}$ lens and with battery loaded it weights $2.6 \mathrm{~kg}$. This camera has temperature accuracy of $\pm 2^{\circ}$.

This camera uses infrared thermography method to detect 


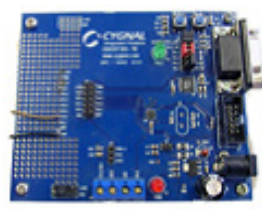

(a) $\mathrm{C8051}$

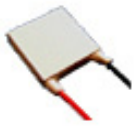

(c) Peltier device

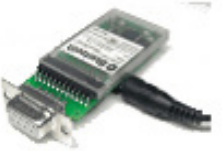

(b) Bluetooth module

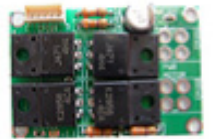

(d) Driver circuit

Fig. 4. Compornents of the Thermo-Landmark.

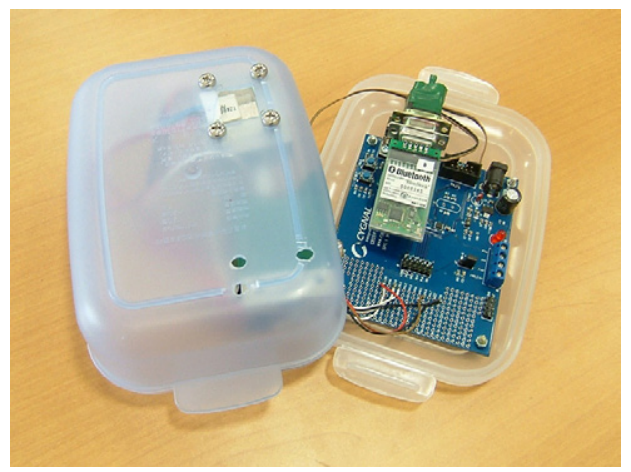

Fig. 5. Developed Thermo-Landmark Unit.

infrared energy emitted from objects surfaces and converts it to temperature. According to the intensity received, a 256 step gray scale image is created. Camera has a field of vision of $30^{\circ}$ from the horizontal and $24^{\circ}$ from the vertical. Image resolution is of $340 \times 240$ pixels. Output images are in NTSC signal mode. Temperature images are sent to our image processing module "SuperIPcam". Camera is connected by serial port to the SuperIPcam which is connected to the user PC by LAN (for reference see Fig.2).

\section{Mobile Robot}

We use a Yamabico Platform robot, which is a small sized mobile robot with two independent wheels and two free casters. The robot size is of $51.6 \mathrm{~cm}$ height, $40 \mathrm{~cm}$ length and $40 \mathrm{~cm}$ width. Mobile robot can process odometry and it counts with motion control algorithms.

Robot has a notebook computer on board for own position calculation and motion controlling. This PC is the master controller of the whole system controlling the communication between landmark module and infrared camera.

\section{LANDMARK LOCALIZATION METHOD}

A gray-scale temperature image (shown in Fig.7) from the environment is taken by infrared camera and sent to image processing module (Hitachi Super IPcam). First we

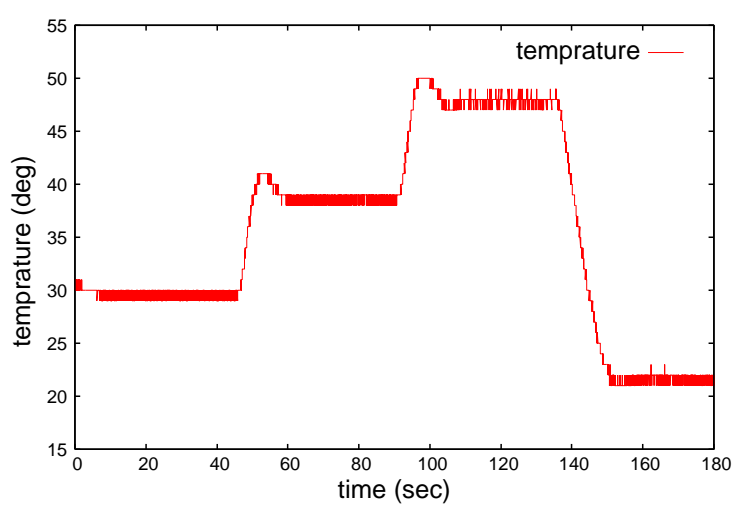

Fig. 6. Thermo responses of a peltier device.

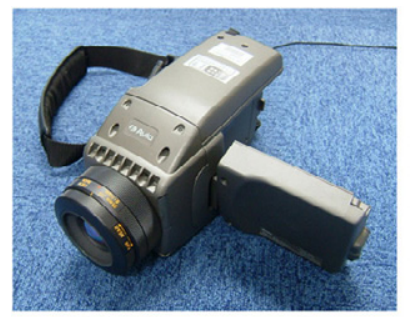

(a) IR camera

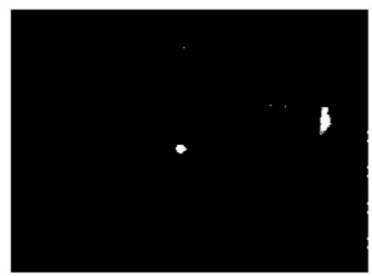

(c) Binarized image

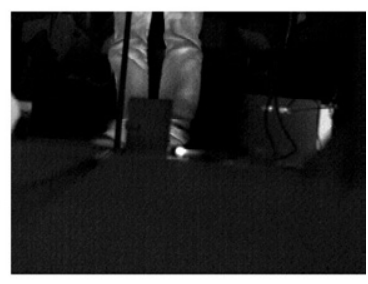

(b) Raw thermo image

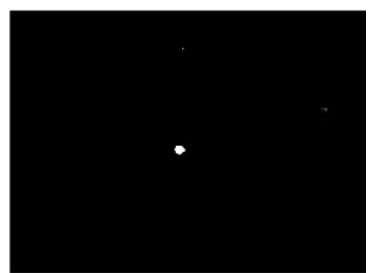

(d) Filtered image
Fig. 7. Thermo camera and thermo images.

search for the landmark in the image, then we calculate its screen coordinates $(u, v)$, finally, relative global coordinates $(x, y, z)$ in real environment are calculated.

\section{A. Finding Temperature Landmark Screen Coordinates $(u, v)$}

First temperature areas that are different to the landmark temperature are excluded. Then noise removal process if performed in order to extract only the landmark. Concretely the process applied to the gray scale image is: 1 . Temperature field extraction (area binarization). 2.Area filtering. 3. Shape filtering. 4. Finally, calculation of center of gravity to the remaining area is done.

Image processing is performed with SuperIPCam by hardware, so the total process of 1 frame is beneath 30milliseconds.

1) Temperature Field Extraction: Infrared Camera takes gray-scaled temperature images while it asks for the actual temperature of the landmark $\alpha^{\circ}$ in order to search for it in the proximities of this temperature. Because 
of environmental temperature influence, areas far from the mark gradually change its temperature between mark area to environmental temperature where $\beta=\alpha-$ (EnvironmentalTemperature). Concretely binarization is performed on pixels with temperatures between $T_{m} a x$ and $T_{m}$ in found with next expressions:

$$
\begin{aligned}
& T_{\max }=\alpha-0.02 \beta+1.8 \\
& T_{\min }=\alpha-0.12 \beta-1.4
\end{aligned}
$$

where, $\beta$ is difference of between mark's and room's temperature. After this process, objects with big temperature difference to the landmark can be eliminated.

2) Area Filtering: After temperature filtering, there are still some objects with similar temperature to the mark. In this case, area filtering is needed. The exposed area of landmark is of $15 \mathrm{mmx} 15 \mathrm{~mm}$ and has a square shape. This mark can be captured by infrared camera from 1 to 3 meters of distance. In order to find landmark areas smaller than the area of the mark captured at 3 meters and areas bigger than the area of the mark captured at the minimum distance of 1 meter are excluded.

3) Shape Filtering: Because of temperature gradation next to the landmark, a line area might remain after area filtering. So, from remaining areas in the image, if the ratio between major and minor axis is below 0.5 or over 2 then these areas are removed.

4) Temperature Re-Setting: In the case landmark was not found or there were more than one probable areas found as possible landmarks, camera module sends an order to landmark module to modify its temperature. Temperature histogram from thermo image taken from camera is analyzed (see Fig.8), then less common temperature range is selected and sent to landmark.

In this particular example it can be seen that most objects have a temperature between $15^{\circ}-25^{\circ} \mathrm{C}$ and $35^{\circ}-38^{\circ} \mathrm{C}$. There are almost no objects with different temperature ranges. In such a case, our temperature landmark module can be ordered to modify its temperature to the less common or non existing temperatures, such as the ones shown in the gaps of the histogram.

This action is repeated constantly so that the landmark can change its temperature autonomously in order to be tracked by the infrared thermograph camera.

\section{B. Relative Distance between Camera and Mark}

After filtering process, the remaining area is considered to be the landmark. Coordinates of center of mass are calculated.

¿From calculated position of landmark on screen coordinates $(u, v)$, relative position to the robot in a global coordinate system cannot be calculated directly. For this reason in this research we assume the condition that height $Z$ of the camera on the robot to the horizontal is fixed and does not change.

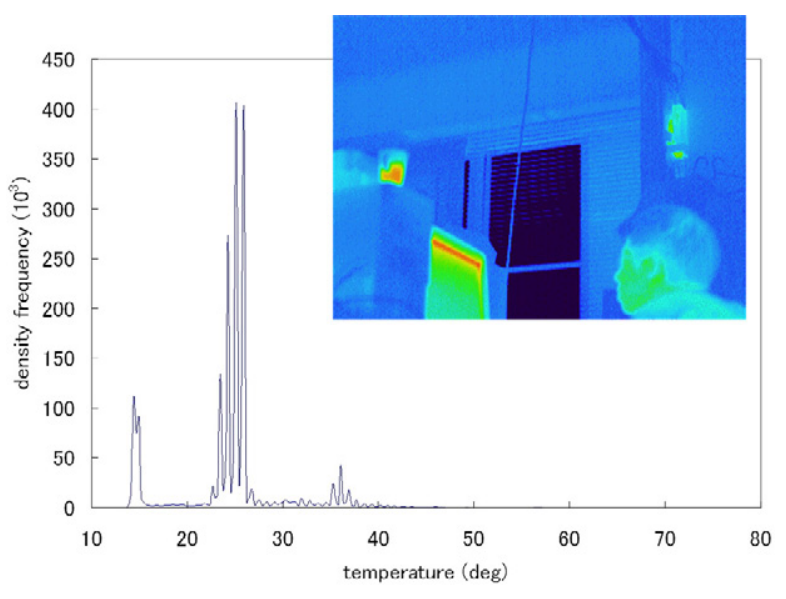

Fig. 8. Thermo Histogram taken from Temperature Image

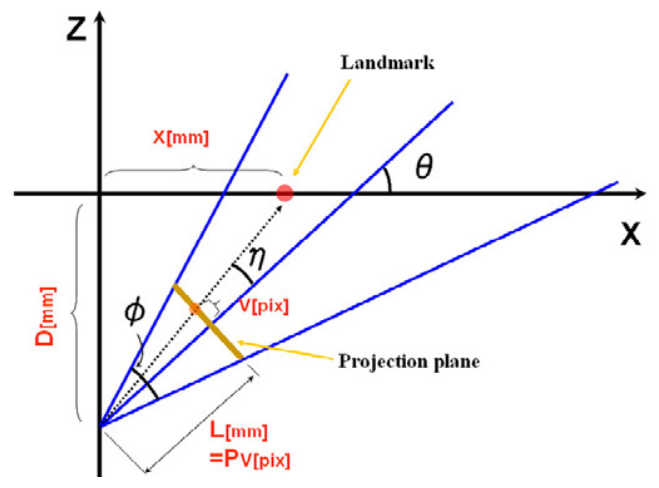

Fig. 9. Camera Coordinates.

Based on this assumption, only coordinates $(x, y)$ have to be calculated. As shown in Fig.9, camera orientation is $\theta$ degrees. The distance between landmark and camera is $D$ centimeters with the assumption that height is constant. Then coordinates $x, y$ can be easily calculated with the next expression.

$$
\begin{gathered}
x=\frac{D}{\tan \left(\theta+\arctan \frac{v}{P_{v}}\right)} \\
y=\frac{x u}{P_{u}}
\end{gathered}
$$

where $P_{u}, P_{v}$ are the projection from lens focus to the plane of projection in horizontal and vertical direction in pixel size.

\section{VERIFICATION EXPERIMENTS}

\section{A. Mark Detection and Position Estimation}

In order to verify the utility of the explained system, landmark position estimation experiments were done. In experiments presented in this section, thermo camera was placed on the mobile robot with an inclination of $20^{\circ}$ over the horizontal and the landmark was placed at a height of $64 \mathrm{~cm}$. In this experiment, position of the landmark was estimated 


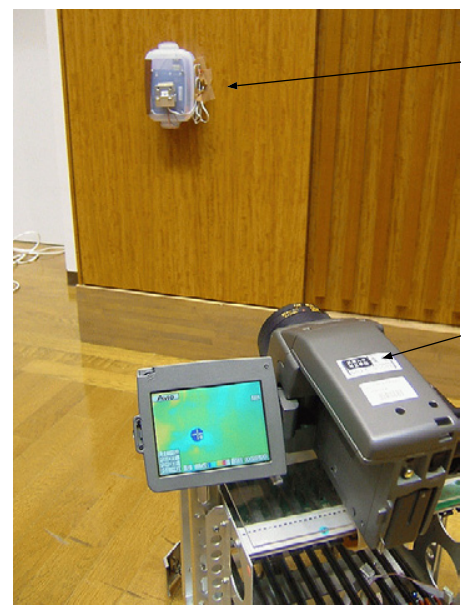

Thermo-Landmark

IR thermo camera and mobile robot

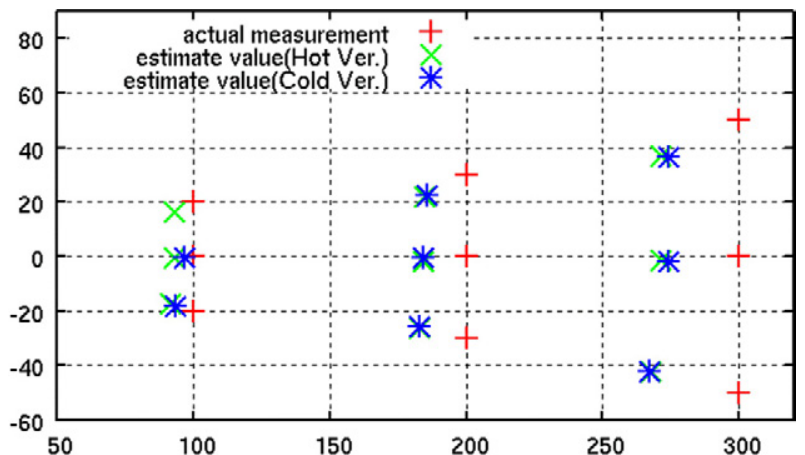

Fig. 11. The Recognition Result of Landmark Position.

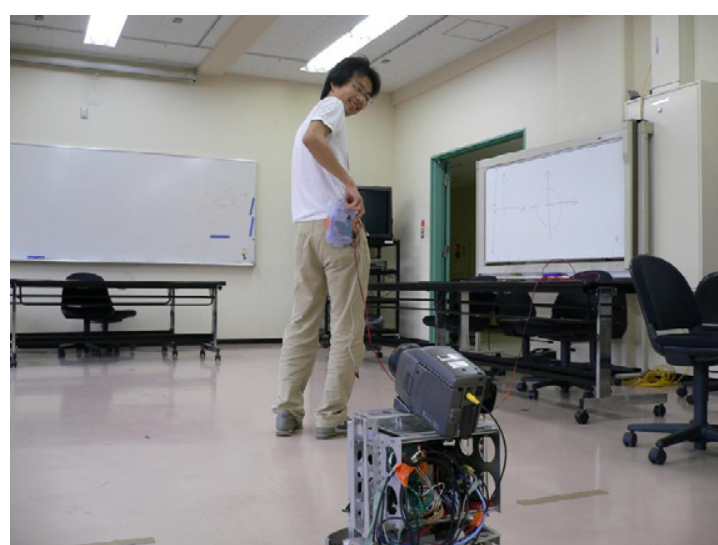

Fig. 12. A Scene of Human Following.

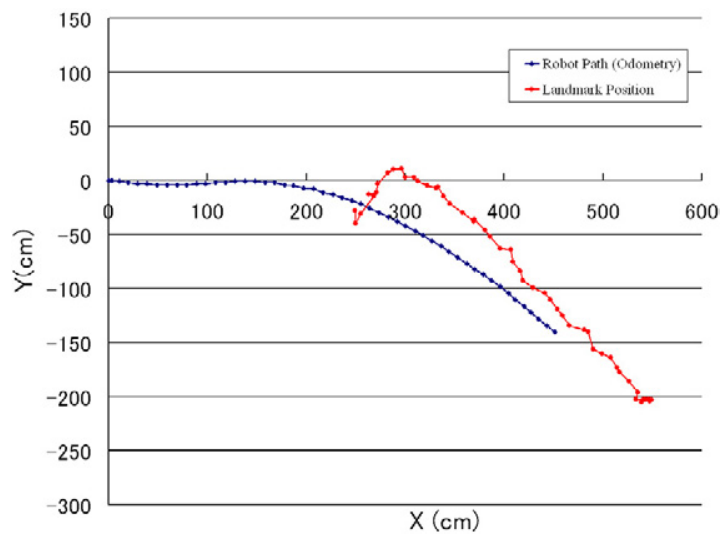

Fig. 13. Human Following Trajectory. when landmark was located to a minimum distance of 1 meter to a maximum distance of 3 meters to the camera while moving to the right and left sides of it. Environment temperature was $25^{\circ} \mathrm{C}$ and landmark temperature was set to a high temperature of $45^{\circ} \mathrm{C}$ and to a low temperature of $15^{\circ} \mathrm{C}$.

Experiment conditions can be seen on Fig.10 and results on Fig.11. In results graph, red mark + represents the real position of the mark and green mark $x$ and blue mark $*$ represents the estimated position of the landmark (green mark is estimated position at high temperature of $45^{\circ} \mathrm{C}$ and blue mark is estimation at low temperature of ${ }^{\circ} \mathrm{C}$ ). At long distance of 3 meters, mark position estimation result was within $30 \mathrm{~cm}$ of the real value, and at $1 \mathrm{~m}$ distance, position estimation accuracy was within $10 \mathrm{~cm}$. In total, position estimation accuracy lies within $5 \%$ of the total distance measured. It is left as future work to increase the accuracy of position estimation.

\section{B. Person Following Application}

To test the effectiveness and robustness of our discreet temperature landmark module, it was attached on a person's belt (approximately $105 \mathrm{~cm}$ from the floor), the thermo camera module is mounted on a mobile robot with the same conditions as previous experiment. The selected environment was a common meeting hall which has common objects such as tables and chairs. When the particular landmark is localized, its position is estimated and the mobile robot starts tracking and moving towards it while following the person. Landmark estimated position located on user's belt and robot followed path are shown in Fig.13. Estimated path followed by user of landmark is showed in blue and path followed by robot calculated by odometry in red. It can be seen in the graph how mobile robot follows a person having the temperature mark moving in zigzag. Our temperature landmark could be found and tracked even in the presence of other obstacles. Our method proved to be robust for finding landmark in a common indoor environment with multiple objects without the utilization of people walking models [11][12], which would not be robust in the case that tracked person does not show both feet.

\section{CONCLUSIONS}

In this paper we presented, explained and showed in experiments our proposal of a changeable discreet landmark which possesses unique features even in dynamic complex indoor environments. In our system we explained how a landmark could actively change its own features according to the purpose it is needed (hide in the environment adjusting tem- 
perature to its surroundings or showing unique characteristics changing to a different temperature) and also to its surrounding conditions. We constructed a landmark module using a peltier device which can change the temperature properties of its body. This device by changing its temperature in a determined environment could be found by a thermo infrared camera sensor. Moreover experiments showed landmark position estimation with accuracy within $5 \%$. Finally a person following application experiment showed the effectiveness of our proposed system.

\section{FUTURE WORKS}

We consider that it is difficult to find environments in which a suitable temperature can not be found (environments where there are very hot and cold temperatures at the same time), however, if robot is located at such environment, as future work we consider the use of two temperature landmarks placed one next to each other with different temperatures (which could be different for 30 or 40 degrees). We consider that robust landmark tracking could be achieve because of its caracteristics of size, form and temperature.

Moreover, present authors consider the case of implementing a system using multiple modifiable landmarks in the environment for robot localization.

\section{ACKNOWLEDGMENTS}

The authors would like to thank to A. Nakajima, T. Shimizu, H. Sekiguchi and M. Takahata for their assistance on this research.

\section{REFERENCES}

[1] Feng Lu and Evangelos Milios

"Robot Pose Estimation in Unknown Environments by Matching 2D Range Scans"

Journal of Intelligent and Robotic Systems, Volume 18, Issue 3 (1997) 249-275.

[2] Feng Lu and Evangelos Milios

"Globally Consistent Range Scan Alignment for Environment Mapping"

Autonomous Robots, Volume 4, Issue 4 (1997) 333-349.

[3] Jason L. Hill, David E. Culler :

"MICA: A WIRELESS PLATFORM FOR DEEPLY EMBEDDED NETWORKS",

IEEE Micro, Vol. 22, pp. 12-24. 2002.

[4] Tsutomu Takeshita, T. Tomizawa and Akihisa Ohya

"A House Cleaning Robot System -Path indication and Position estimation using ceiling camera-"

SICE-ICASE International Joint Conference 2006, Busan(Korea), 2006.

[5] Heeseong Chae, Jaeyeong Lee and Wonpil Yu

"A Localization Sensor Suite for Development of Robotic Location Sensing Network"

The 2nd International Conference on Ubiquitous Robots and Ambient Intelligence Intelligent Robot Division, ETRI 161, 2005.

[6] Akihisa Ohya, Yousuke Nagumo and Youhei Gibo

"Intelligent Escort Robot Moving together with Human -Methods for Human Position Recognition-",

Joint 1st International Conference on Soft Computing and Intelligent Systems and 3rd International Symposium on Advanced Intelligent Systems (SCIS \& ISIS 2002), 24B5-2, Oct. 2002.

[7] K. Takemura, K. Ohara, K. Ohba, N. Y. Chong, S. Hirai, K. Tanie, "Knowledge Distributed Tag-Based Vision System,"

Proceedings of Int. Workshop on Networked Sensing Systems, pp. 179-182, Tokyo, 2004.
[8] Kazunori Ohno, Takashi Tsubouchi, Bunji Shigematsu and Shin'ichi Yuta

"Mobile Robot Navigation Based on DGPS and Odometry in Campus Environment"

Proc. of IEEE Int Conf. on Field and Service Robotics (2003) 189-194.

[9] Petrovski I., K. Okano, M. Ishii, H. Torimoto, Y. Konishi and R. Shibasaki

"Pseudolite implementation for social infrastructure and seamless Indoor/Outdoor positioning." ION GPS 2002, 69-76.

[10] Yusuke Nakazato, Masayuki Kanbara and Naokazu Yokoya "Discreet Markers for User Localization"

Proceedings of the Eighth International Symposium on Wearable Computers (ISWC'04)1530-0811/04.

[11] H. Zhao and R. Shibasaki

"A Novel System for Tracking Pedestrians Using Multiple Single-Row Laser-Range Scanners,"

IEEE Transactions on Systems, Man, and Cybernetics-Part A, Vol. 35, No. 2, 2005.

[12] J. H. Lee, T. Tsubouchi, K. Yamamoto and S. Egawa

"People Tracking Using a Robot in Motion with Laser Range Finder" Proceedings of the 2006 IEEE/RSJ International Conference on Intelligent Robots and Systems pp.2936-2942 2006, 